\title{
Study on Hybrid Energy Storage Configuration and Control Strategy of Grid-connected Wind Hydrogen System
}

\author{
Xiaojun Shen ${ }^{1, *}$, Congying $\mathrm{Nie}^{1}$, Hong $\mathrm{Lv}^{2}$, Wei $\mathrm{Wang}^{3}$ \\ ${ }^{1}$ Department of Electrical Engineering, Tongji University, Shanghai 201804, China \\ ${ }^{2}$ Automotive College, Tongji University, Shanghai 201804, China \\ ${ }^{3}$ State Grid Corporation, Beijing 100031, China
}

\begin{abstract}
The randomness and volatility of wind energy bring great challenges to wind power grid-connected. The hybrid energy storage technology based on electrolysis cell hydrogen production and super capacitor has become an effective way to stabilize wind power fluctuations. Based on the establishment of the grid-type wind-hydrogen coupling system working characteristic constraint and the minimum initial investment cost of the hybrid energy storage system, an alkaline electrolytic cell-super capacitor hybrid storage based on low-pass filtering-fluctuation observation is proposed. The method can be configured, and a hybrid energy storage coordinated control strategy based on the super capacitor SOC (state of charge) is developed. The case study results show that the hybrid energy storage system configuration method and control strategy proposed in this paper are effective, which can reduce the fluctuation of wind power grid-connected power to meet the grid connection standard.
\end{abstract}

\section{Introduction}

Wind power is the main form of utilization of renewable energy. Its rapid and large-scale development makes it difficult for grids to absorb wind power and the problem of abandoning wind power is getting worse. Hydrogen energy is characterized by clean, pollution-free, highly efficient and renewable. It is regarded as the most potential energy carrier in the future. The use of electrical energy generated by renewable energy to electrolyze water to produce hydrogen is considered to be the best way to lead to hydrogen economy [1]. The related theoretical research and engineering demonstration application results show that the combination of electrolyzed water hydrogen production and wind power can effectively stabilize the wind power volatility and enhance the schedulability of

* Corresponding author: xjshen79@163.com 
wind power, and provide a new technical solution for solving wind power consumption problems, which is a research hotspot at home and abroad [2].

The literature search results show that since the beginning of this century, wind power hydrogen production technology has gradually gained worldwide attention, and related research has been carried out. The early wind hydrogen system demonstration project focused on wind power, hydrogen production (electrolysis tank), hydrogen storage and direct simple power control [3]. In recent years, with the rapid development of wind-hydrogen system demonstration projects, research has become more and more in-depth, and rich research results have been achieved in hydrogen production power conversion technology, system coordination control, capacity allocation, program planning and technical economic analysis. For example, the reference [4] proposes a smooth control strategy for multi-type energy storage systems using real-time wavelet transform for frequency allocation and double-layer fuzzy control theory for energy storage power correction. In reference [5], the spectrum analysis method is used to optimize the energy storage capacity distribution of the energy storage system, which can effectively reduce the fluctuation of wind power and ensure that the energy storage equipment will not be over flushed or over discharged. In reference [6], the mathematical model of the hybrid system of wind power / photovoltaic / hydrogen production / super capacitor is established, and the corresponding control strategy is proposed for different operation conditions. The operation efficiency of the system is improved, the DC bus voltage is stable, and the operation reliability of the system is guaranteed; The energy storage forms used in reference [7] are electrolytic cell, hydrogen storage tank and proton exchange membrane fuel cell cycle system, which are connected to the DC side of the power converter of the wind power system to form the wind power hydrogen cogeneration system, and the coordinated control strategy of each component unit is designed. The simulation results show that the proposed control strategy is effective; In reference [8], based on the DC coupled wind, hydrogen and super capacitor hybrid power system, two power management strategies are proposed and compared through experiments. It is found that the "source tracking" strategy has better performance than the "grid connected" strategy in power grid regulation. In reference [9], based on the analysis of the spectrum characteristics of wind power, the wavelet packet decomposition technology is used to decompose it into low-frequency grid connected part and high-frequency part, and then the probability density function of fluctuating power is fitted by Fourier approximation to obtain the expected energy storage capacity configuration and optimize the energy storage capacity. In reference [10], taking the maximum profit of the system as the objective, considering environmental benefits, government subsidies and time value of funds, the objective function and its constraints are established, and the economic evaluation is carried out by using the improved chemical reaction algorithm. However, the capacity of hydrogen production and fuel cell cannot 
completely smooth the fluctuation of wind power in the maximum profit, and other energy storage equipment needs to be added.

The results of literature analysis show that the existing research results consider the characteristics of electrolytic cell less, which is not conducive to engineering application. In view of this, based on the study of the wind hydrogen coupling system and the actual external characteristics of the alkaline electrolyzer, the configuration method and process of the electrolyzer super capacitor hybrid energy storage system are established based on the low-pass filter volatility observation method, and the optimal coordination control strategy is formulated. Finally, the effectiveness of the configuration method and control strategy is verified by the case simulation.

\section{Topology and constraints of grid connected wind hydrogen coupling system}

\subsection{Overview of grid connected wind hydrogen coupling system}

Wind hydrogen coupling power generation has become an important means to solve the "bottleneck" problem of wind power connected to grid. As a kind of clean energy, hydrogen can feed back electric energy to the power grid through hydrogen fuel cell in the low period of power generation, or enter the industrial and commercial fields through vehicle or pipeline. At the same time, the wind hydrogen coupling system will greatly promote the development of hydrogen fuel cell vehicle industry rapidly [11]. The typical wind hydrogen coupling hybrid energy storage system includes wind turbine, electrolytic water hydrogen generation unit and super capacitor energy storage unit, as shown in Figure 1.

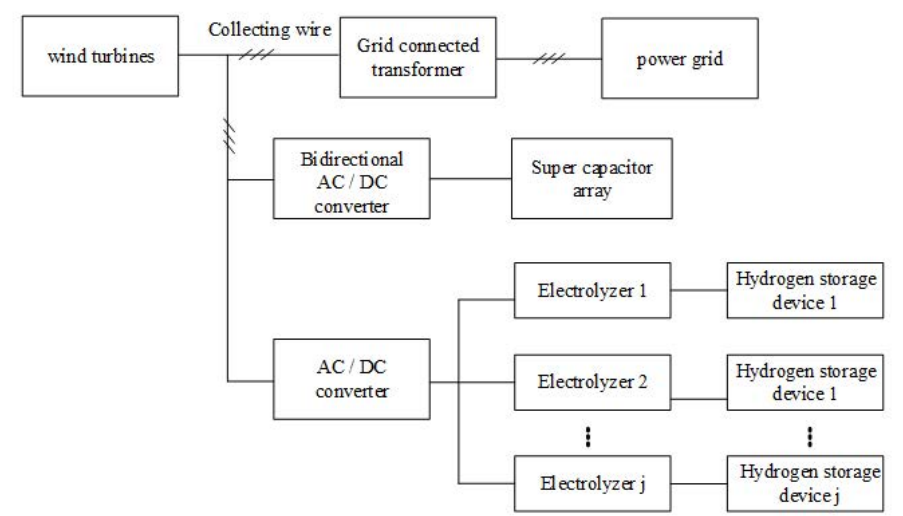

Fig.1. The structural diagram of the wind-hydrogen coupling system

The electrolytic cell and super capacitor are connected with the wind power system through AC / DC device, in which the electrolytic cell is the main energy storage equipment as the cost of it is low, but due to the lack of absorption for high-frequency power, super 
capacitor is introduced as the auxiliary energy storage equipment [12].

\section{2 working characteristics constraints of wind hydrogen coupling system}

\subsection{1 working characteristics constraints of electrolyzer}

According to the relevant research results of the external characteristics of the alkaline electrolyzer, the external characteristics of the electrolyzer can be expressed as [13]:

$$
\left\{\begin{array}{l}
V \in\left[V_{\min }, V_{\max }\right] \\
T \in\left[T_{\min }, T_{\max }\right] \\
I \in\left[I_{\min }, I_{\max }\right] \\
P \in\left[P_{\min }, P_{\max }\right] \\
\Delta t \leq 1 m s
\end{array}\right.
$$

Where, $\mathrm{V}$ is the working voltage; I is the working current, and the range corresponds to the voltage; $\mathrm{T}$ is the working temperature; $\Delta t$ is the response time; $\mathrm{P}$ is the working power, and its lower limit value is not lower than the thermal insulation power $P_{\text {min }}$. Thermal insulation power is defined as the balance of heat production and heat dissipation of the electrolyzer under this power condition, and the temperature stability of the electrolyzer can be guaranteed without external environmental control device. Only when the power of the cell is not lower than this value can the cell have a millisecond level response speed.

\subsection{2 working characteristics constraints of super capacitor}

As a kind of energy storage equipment with excellent properties, super capacitor has the characteristics of fast response and high efficiency. However, the biggest disadvantage of limiting its application is its high price, and its energy unit price is about 30 times of the traditional lithium-ion battery [14]. In consideration of the economy, the super capacitor, as an auxiliary energy storage device, generally occupies a small part of the capacity in the hybrid energy storage system. In general, super capacitor exists in the form of modularization, through different number of modules in series or parallel to get the corresponding demand energy storage system. In the engineering application of super capacitor, the constraint of formula (2) should be satisfied.

$$
\left\{\begin{array}{l}
E<E_{\max } \\
U<U_{N} \\
I<I_{\max } \\
S O C \in\left[S O C_{\min }, S O C_{\max }\right]
\end{array}\right.
$$

Where $E_{\max }$ is the maximum value of stored energy; $U_{N}$ is the rated voltage; $I_{\max }$ 
is the maximum value of allowable passing current; SOC is the state of charge of super capacitor. In order to ensure the normal and efficient operation of super capacitors and good service life, SOC of general super capacitors has a certain range of constraints.

\subsection{3 characteristics constraints of wind turbine and grid connection}

Without considering the wake and electrical losses of the wind farm, the output power of the wind farm is equal to the sum of the output of all the fans in the field, and the power output of the fans mainly depends on the wind speed at the height of the fan hub. The relationship between the two can be expressed by the following subsection function [15]:

$$
P_{w}=\left\{\begin{array}{cc}
0 & V<V_{\text {in }} \text { 或 } V_{\text {out }}<V \\
\frac{P_{e}\left(V^{3}-V_{\text {in }}^{3}\right)}{V_{\text {out }}^{3}-V_{\text {in }}^{3}} & V_{\text {in }}<V<V_{e} \\
P_{e} & V>V_{e}
\end{array}\right.
$$

Where $V$ is the wind speed at the wind turbine; $V_{\text {in }}$ is the cut-in wind speed; $V_{\text {out }}$ is the cut-out wind speed; $V_{e}$ is the rated wind speed; $P_{e}$ is the rated output power; $P_{w}$ is the actual output power of the wind turbine.

In order to ensure the safety and reliability of wind power connected to grid, all countries have developed corresponding technical indicators for wind power connected to grid. China has also formulated relevant regulations for the grid connection of wind farms with different installed capacity, and the maximum change of output active power of wind farms must be within the maximum change limit of $1 \mathrm{~min}$ and $10 \mathrm{~min}$ active power [16].

$$
\left|P_{\text {wind }}-P_{e l}-P_{s c}\right| \leq P_{\max }
$$

In the formula, $P_{\text {wind }}$ is the original power of wind farm; $P_{e l}$ is the absorption power of electrolytic cell; $P_{s c}$ is the absorption power of super capacitor; $P_{\max }$ is the maximum limit value of power fluctuation specified in national standard, and there are two different values on the time scale of $1 \mathrm{~min}$ and $10 \mathrm{~min}$.

Table 1. Maximum limit of wind farm active power change

\begin{tabular}{ccc}
\hline $\begin{array}{c}\text { Installed capacity } \\
\text { of wind farm (MW) }\end{array}$ & $\begin{array}{c}\text { 10min active power } \\
\text { Change value (MW) }\end{array}$ & $\begin{array}{c}\text { 1min active power } \\
\text { Change value (MW) }\end{array}$ \\
\hline$<30$ & 10 & 3 \\
$30 \sim 150$ & Installed capacity/3 & Installed capacity/10 \\
$>150$ & 50 & 15 \\
\hline
\end{tabular}

\subsection{Minimum initial investment cost constraint}

In this paper, the main goal is to stabilize the power fluctuation of wind power by 
electrolyzer and super capacitor. Under this condition, the economy of the hybrid energy storage equipment is considered. Therefore, the profit of hydrogen production is not considered in the evaluation of the economy index, and the initial investment cost of the system is minimized to optimize the configuration scheme.

Investment cost of hydrogen production equipment [17]:

$$
F_{E L}=P_{E L} u
$$

In the formula, $u$ is the construction cost of hydrogen production equipment per unit capacity; $P_{E L}$ is the power of the electrolytic cell.

The investment cost of super capacitor can be determined according to the capacity [14]:

$$
F_{S C}=W_{S C} f_{n}
$$

In the formula, $W_{S C}$ is the capacity of the super capacitor; $f_{n}$ is the capacity unit price of the super capacitor.

According to the market of super capacitor and electrolyzer, the cost of super capacitor is much higher than that of electrolyzer when the same amount of electricity is stored. Therefore, in order to minimize the cost of the hybrid energy storage system, the electrolyzer is preferred as the main energy storage equipment and the super capacitor as the auxiliary energy storage equipment. At the same time, because of the particularity of the electrolyzer, the negative value of the power fluctuation, that is to say, the energy storage system needs to provide power to the grid, which must be borne by the super capacitor, which requires that the capacity of the super capacitor cannot be lower than a certain limit value. In order to make the system meet the grid connection standard and consider the economy, it is necessary to find out the minimum limit value of super capacity and set its capacity as this value.

$$
\left\{\begin{array}{l}
W_{S C}=W_{S C \text { min }} \\
F_{\text {min }}=F_{E L \text { max }}+F_{S C \text { min }}
\end{array}\right.
$$

Where, $W_{S C \min }$ represents the minimum capacity of the super capacitor that meets the conditions; $F_{\min }$ represents the minimum total cost of the initial investment of the system; $F_{E L \max }$ represents the maximum cost of the corresponding electrolyzer, and $F_{S C \min }$ represents the minimum cost of the super capacitor.

\section{Configuration of hybrid energy storage system based on low pass filter and fluctuation observation}

The configuration roadmap of the wind power fluctuating energy storage system adopted in 
this paper is shown in Figure 2. First, the original wind power to be suppressed is divided into the allowable fluctuating part and the exceeding part according to the national grid connection standard by algorithm; then, the exceeding part is further divided into low frequency component and high frequency component by spectrum analysis, and the low frequency component focuses on the energy density and high frequency component focuses on the power density of the energy storage medium. Finally, according to the characteristics of the energy storage medium, the matching selection is carried out to complete the configuration of the mixed energy storage.

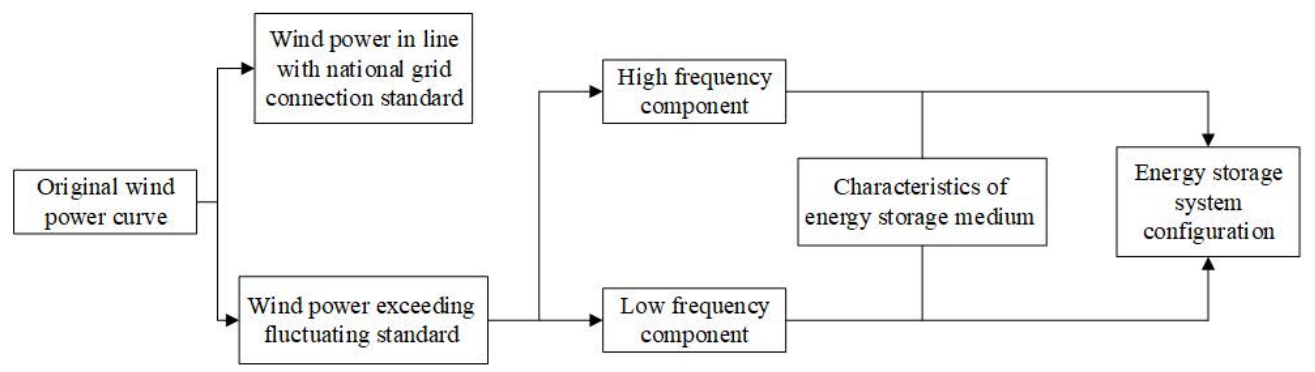

Fig.2. Configuration Technology Roadmap

\subsection{Low pass filtering}

In this paper, the first-order low-pass filtering method is used to analyze the power fluctuation of wind power to be suppressed. The fluctuating power is regarded as a fluctuating signal, so the low-pass filtering is used to filter out the high-frequency components, so as to reduce the power fluctuation. The traditional low-pass filtering methods are as follows [18]:

The first-order low-pass filter method is mainly improved from the first-order low-pass filter, and its mathematical expression is as follows:

$$
\tau * \frac{d U_{o}}{d t}+U_{o}=U_{i}
$$

Where $\tau=R C=1 / 2 \pi f_{c}$ is the time constant of the low-pass filter, and $f_{c}$ is the cut-off frequency of the filter. $U_{o}$ is the output signal and $U_{i}$ is the input signal. When applied to the fluctuation of wind power, the input signal is the original fluctuation power $P_{w}$, and the output signal is the power $P_{o}$ connected to the grid after being flattened. The purpose of this paper is to make the fluctuation of grid connected power $P_{o}$ smaller and meet the corresponding grid connected standards.

Let the period of wind power sampling be $T_{s}$, and discretize formula (8) to obtain:

$$
\tau \frac{P_{o}(t)-P_{o}\left(t-T_{s}\right)}{T_{s}}+P_{o}(t)=P_{w}(t)
$$

From equation (9): 


$$
P_{o}(t)=\frac{\tau}{\tau+T_{s}} P_{o}\left(t-T_{s}\right)+\frac{T_{s}}{\tau+T_{s}} P_{w}(t)
$$

Since the change range of time constant $\tau$ is $[0, \infty)$, it is difficult to optimize the adjustment, so that let $\alpha=T_{s} / \tau+T_{s}, \alpha$ is called the filter coefficient, then equation (10) can be written as:

$$
P_{o}(t)=(1-\alpha) P_{o}\left(t-T_{s}\right)+\alpha P_{w}(t)
$$

It can be seen from equation (11) that the larger the filter coefficient is, the greater the impact of input power on output power is; the smaller the filter coefficient is, the greater the impact of historical value of output power on output power is, the smoother the curve of output power is, and the better the filtering effect is.

\subsection{Window sliding wave observation method}

In order to analyze the fluctuation of power, judge whether it meets the national grid connection standard, and find the best filter coefficient to minimize the initial investment cost of the system. In this paper, a window sliding observation method is proposed to extract data from the power waveform and analyze the data. The specific methods are as follows: firstly, continuous sampling data at $1 \mathrm{~min}$ interval and analysis it. According to the definition of $10 \mathrm{~min}$ wave momentum, it is necessary to collect the power data in the first ten minutes of each time, find the maximum and minimum values of these ten data, take the difference as the maximum value of $10 \mathrm{~min}$ wave momentum at this time and store it in the array. The width of these 10 data is the width $\mathrm{D}$ of the established window. As time goes on, the window also moves together, so that 10 min fluctuation of each time can be obtained.

Set $t_{1}, t_{2}, t_{3} \cdots t_{n}$ is the sampling time, where $\mathrm{n} \in[1,600]$. The corresponding sampling values are $S_{t_{1}}, S_{t_{2}}, S_{t_{3}} \cdots S_{t_{n}}$. For any time $t_{n}(\mathrm{n}>10)$, the fluctuation in 10 minutes is as follows:

$$
A_{t_{n}}=\operatorname{Max}\left(S_{t_{n-10}} \cdots S_{t_{n}}\right)-\operatorname{Min}\left(S_{t_{n-10}} \cdots S_{t_{n}}\right)
$$

Among them, $\operatorname{Max}\left(S_{t_{n-10}} \cdots S_{t_{n}}\right)$ represents the maximum value of data in this time window, $\operatorname{Min}\left(S_{t_{n-10}} \cdots S_{t_{n}}\right)$ represents the minimum value of data in this time window.

In the same way, for 1 min fluctuation, just adjust the window size $\mathrm{D}$ to 2 . Figure 3 is the schematic diagram of the window sliding wave observation method. The red box represents the window size set up. As time goes by, the window moves with it, so that only the data in the window needs to be analyzed to get the required wave fluctuation information. 


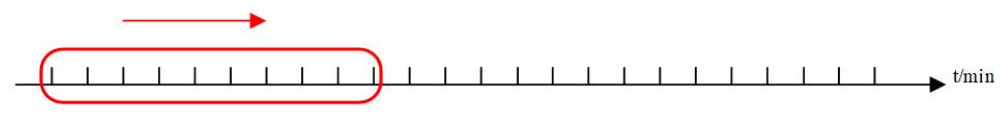

Fig.3. Window sliding schematic diagram

\subsection{Hybrid energy storage capacity allocation process based on low-pass filtering and volatility observation}

The configuration flow of hybrid energy storage system proposed in this paper is shown in Figure 4.

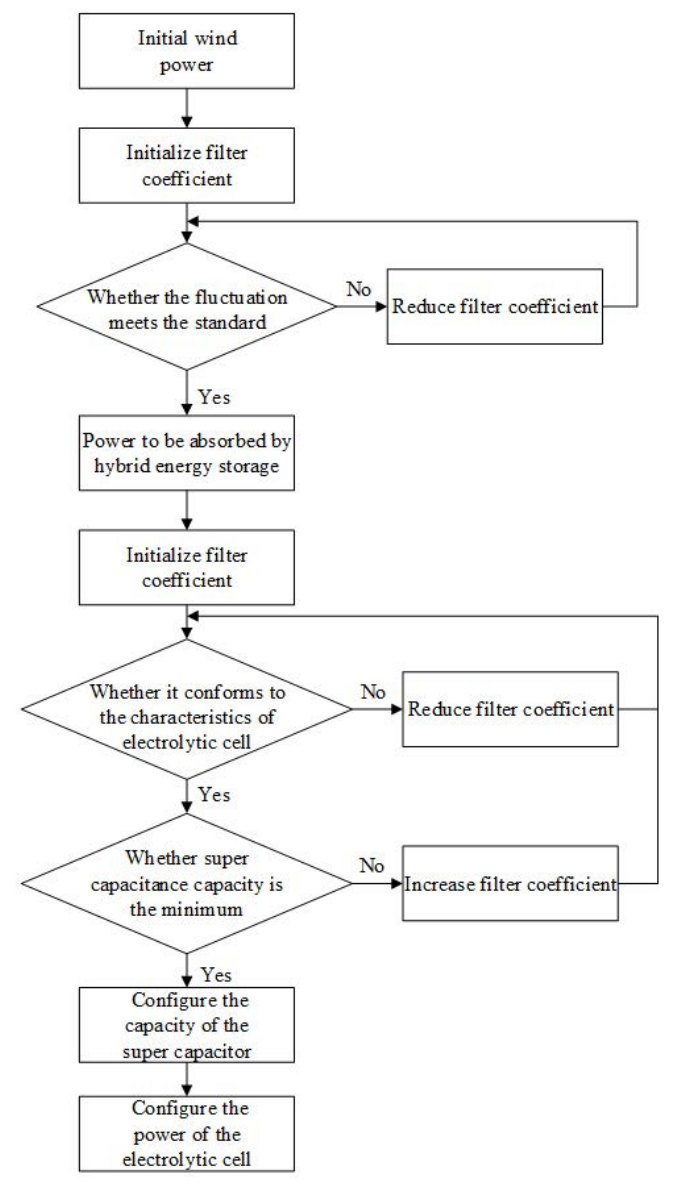

Fig.4. Hybrid energy storage contiguration flow chart

The first step is to distribute wind power based on low-pass filtering. After the first low-pass filtering of the initial wind power, the new grid connected power and the fluctuating power needed to be absorbed by the energy storage system are obtained, the filtering coefficient is determined by seeking the maximum value as far as possible under the condition that the new grid connected power meets the national grid connected 
standard.

The second step is to distribute the absorbed power of each hybrid energy storage device. Based on the low-pass filtering method, the high-frequency fluctuating power absorbed by the super capacitor and the low-frequency fluctuating power absorbed by the electrolytic cell are obtained. The determination of the filtering coefficient needs to be determined according to the characteristics that the electrolytic cell can only absorb power and the minimum capacity of the super capacitor to achieve the minimum initial investment goal.

The third step is to configure hybrid energy storage. According to the determined filter coefficient, the theoretical power absorption curve of the super capacitor is obtained, and the capacity is configured according to the boundary conditions of the super capacitor, and the power of the electrolytic cell is configured considering the coordinated control strategy of hybrid energy storage.

\section{Coordinated control strategy of hybrid energy storage system}

The control strategy between cell and super capacitor mainly focuses on the power distribution between them. Starting from the state of charge of the super capacitor, the power distribution of the electrolytic cell is based on the state of charge [19]:

$$
P_{e l}=\frac{2 P_{\max }}{S O C_{\max }-S O C_{\min }}\left(S O C_{t}-\frac{S O C_{\max }+S O C_{\min }}{2}\right)
$$

Where $P_{\max }$ is the overall real-time power command of the hybrid energy storage system, whose value is greater than zero indicates the energy absorbed by the energy storage system, otherwise indicates the energy released; $P_{e l}$ indicates the power of the electrolyzer; $P_{S C}$ indicates the power of the supercapacitor; $S O C_{t}$ indicates the real-time state of charge of the supercapacitor; $S O C_{\max }$ and $S O C_{\min }$ are the upper and lower limits of the state of charge of the super capacitor respectively.

It can be seen from equation (13) that when the state of charge of the super capacitor is more than half, the electrolytic cell starts to work; and when the state of charge of the super capacitor is close to the upper limit value, all the power is absorbed by the electrolytic cell; especially, because the electrolytic cell cannot output power, when the $P_{\max }$ is negative, all the power is provided by the super capacitor. The multi condition control strategy of hybrid energy storage system is as follows:

Case 1: when the energy storage system absorbs energy and the state of charge of the super capacitor is greater than $50 \%$, that is $P_{\max }>0$, SOC $>50 \%$. 


$$
\left\{\begin{array}{l}
P_{e l}=\frac{2 P_{\max }}{S O C_{\max }-S O C_{\min }}\left(S O C_{t}-\frac{S O C_{\max }+S O C_{\min }}{2}\right) \\
P_{S C}=P_{\max }-P_{e l}
\end{array}\right.
$$

Case 2: the energy storage system absorbs energy, and the state of charge of the super capacitor is less than $50 \%$, that is $P_{\max }>0$, SOC $<50 \%$.

$$
\left\{\begin{array}{l}
P_{e l}=P_{T \text { min }} \\
P_{S C}=P_{\max }-P_{T \text { min }}
\end{array}\right.
$$

Case 3: the energy storage system release energy, that is $P_{\max }<0$.

$$
\left\{\begin{array}{l}
P_{e l}=P_{T \text { min }} \\
P_{S C}=P_{\max }-P_{T \text { min }}
\end{array}\right.
$$

In case 2 and case 3, the electrolyzer works under the condition of thermal insulation power $P_{\text {Tmin }}$, while in case 2 , the positive value of $P_{s c}$ indicates that the super capacitor absorbs power from the grid, while in case 3, the negative value of $P_{s c}$ indicates that the super capacitor releases power to the grid, so their physical meanings are different.

The transfer of three working conditions is shown in Figure 5.

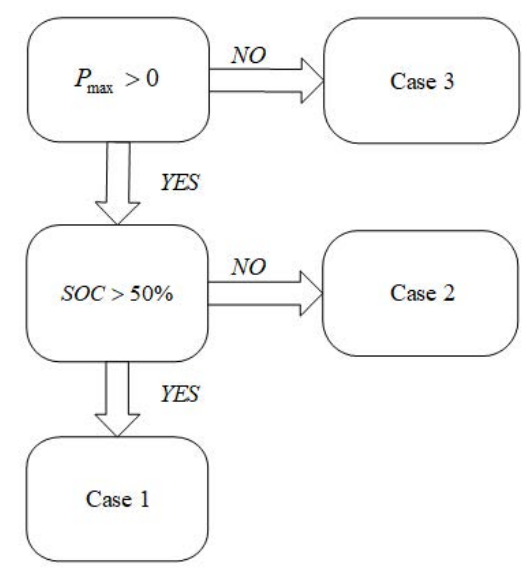

Fig.5. Working condition transfer diagram

\section{Case analysis}

\subsection{Simulation parameter setting and wind power waveform}

In order to verify the effectiveness of the proposed method, this paper takes 50MW wind farm as an example for hybrid energy storage configuration. Based on MATLAB / Simulink, build wind speed model [20] and wind turbine model [21], and the simulation parameters are shown in Table 2. 
Table 2. Simulation parameters

\begin{tabular}{llll}
\hline $\boldsymbol{V}_{\boldsymbol{b}}(\mathrm{m} / \mathrm{s})$ & 4 & $\boldsymbol{\rho}\left(\mathrm{kg} / \boldsymbol{m}^{\mathbf{3}}\right)$ & 1.225 \\
\hline $\boldsymbol{V}_{\boldsymbol{g} \boldsymbol{m a x}}(\mathrm{m} / \mathrm{s})$ & 5 & $C_{p}(\lambda, \beta)$ & 0.45 \\
\hline $\boldsymbol{V}_{\boldsymbol{r} \boldsymbol{m a x}}(\mathrm{m} / \mathrm{s})$ & 2 & $\mathrm{R}(\mathrm{m})$ & 43.5 \\
\hline
\end{tabular}

Where $V_{b}$ is the basic wind, $V_{\text {gmax }}$ is the gust, $V_{\text {rmax }}$ is the gradual wind, $\rho$ is the air density, $C_{p}$ is the wind energy utilization rate, and $\mathrm{R}$ is the radius of the wind turbine. The wind speed simulation model is divided into basic wind, gust, gradual wind and random wind. The accumulation of four kinds of wind can get the natural wind. After the natural wind passes through the wind turbine model, the original wind power is obtained. Through simulation, the wind speed waveform and corresponding power waveform of $10 \mathrm{~h}$ can be obtained as shown in Figure 6.

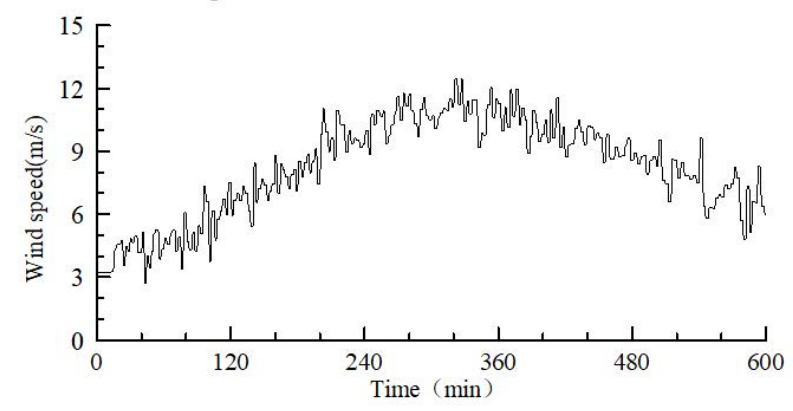

(a) Wind speed waveform

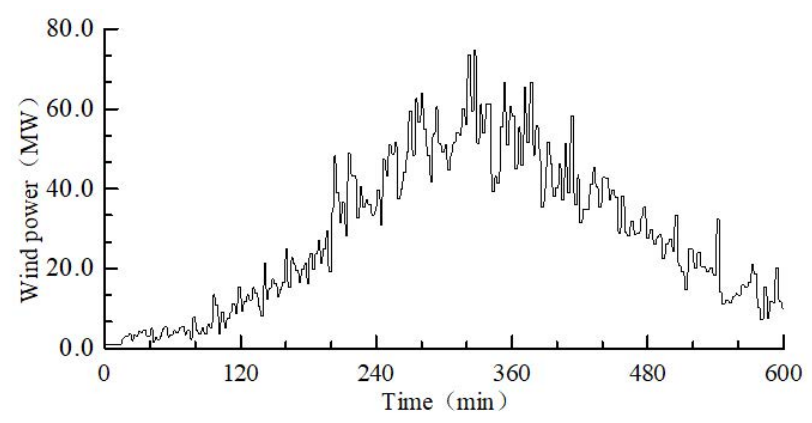

(b) Wind power waveform

Fig.6. Simulated wind speed and wind power curve

\subsection{Analysis of original wind power fluctuation}

Figure 6 (b) is the original wind power curve. Based on the window sliding data processing method, we can get the dispersion diagram of the amplitude and time of the fluctuation in $1 \mathrm{~min}$ and 10min, as shown in Figure 7. 


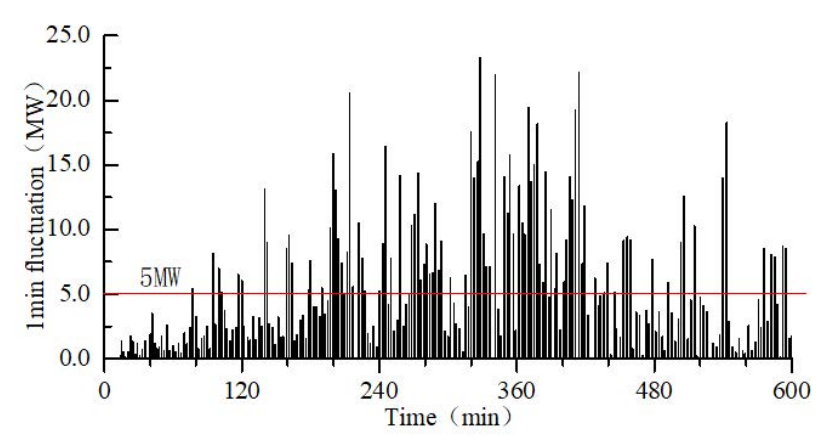

(a) 1 min fluctuation without energy storage system

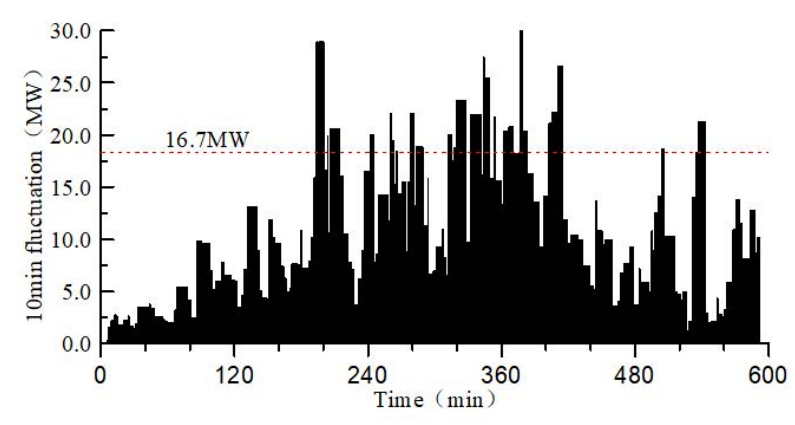

(b) $10 \mathrm{~min}$ fluctuation without energy storage system

Fig.7. Fluctuation without the energy storage system

The red line in Figure 7 is the maximum fluctuation value allowed by the national standard, in which the maximum allowable fluctuation value of $1 \mathrm{~min}$ is $5 \mathrm{MW}$, and the maximum allowable fluctuation value of $10 \mathrm{~min}$ is $16.7 \mathrm{mw}$. Obviously, most of the fluctuation of wind power exceeds the standard before the energy storage system is added.

\subsection{Hybrid energy storage power and capacity economic configuration}

When the original wind power is filtered for the first time, and the filter coefficient is adjusted gradually to make the filtered grid connected power meet the national grid connected standard, then the power consumed by the energy storage system can be obtained. In order to determine the power and capacity of different energy storage equipment, the second low-pass filtering is carried out for the power that needs to be absorbed by the energy storage system, and the theoretical absorption power curve of the super capacitor is obtained. Then the capacity of the electrolytic cell is obtained by the control strategy. Under the condition of ensuring the system's stabilizing ability, the larger the capacity of the electrolytic cell is, the lower the initial investment cost of the system is. That is to say, the absorption power curve of the electrolytic cell is just positive by adjusting the second filter 
coefficient. At this time, the power absorption curve of the super capacitor is shown in Figure 8, and the capacity of the super capacitor configured is the minimum value $W_{S C m i n}$, and the corresponding cost of the super capacitor is the minimum $F_{S C \min }$.

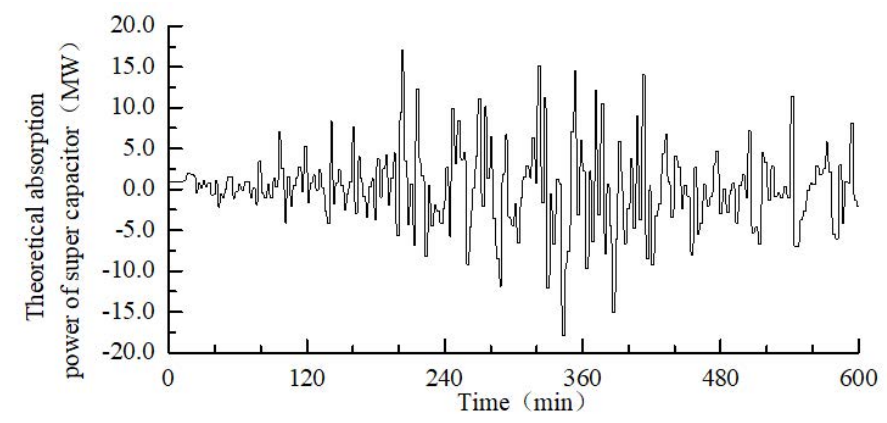

Fig.8. Theoretical absorption power waveform of super capacitor

In this paper, a discrete energy calculation method is used to determine the capacity allocation of the super capacitor. Firstly, sample and process the sample data in Fig. 8, and when $t_{k}=\mathrm{kt}(\mathrm{k}=1,2,3 \cdots \mathrm{n})$, calculate the real-time energy value $E_{s c}$ in kwh according to equation (17).

$$
E_{S C}\left(t_{k}\right)=E_{S C}\left(t_{k-1}\right)+\frac{P_{S C}\left(t_{k}\right)}{3.6 \times 10^{6}}
$$

After the real-time energy data value is obtained according to equation (17), the power change curve of the super capacitor in the whole process can be drawn, from which the maximum value and the minimum value can be found, and then the super capacitor capacity to be configured can be obtained. Considering the charge state range of the super capacitor is $0.05 \sim 0.95$, the final configuration capacity can be obtained by dividing the difference value by 0.9 .

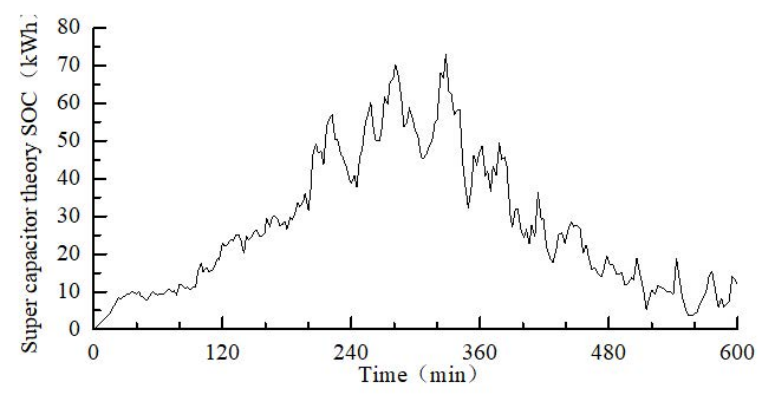

Fig.9. Super capacitor theory energy change value

According to figure 9, the difference between the maximum and minimum value is $72.95 \mathrm{kwh}$. Considering the range of state of charge, the capacity is finally configured as 81.06kwh. After determining the capacity of the super capacitor, considering the 
coordinated control strategy between different energy storage systems, the configuration of the electrolytic cell is as follows:

According to the above control strategy, the super capacitor is charged first, and the absorption power of the electrolytic cell is determined according to the change of the state of charge, so as to obtain the power absorption curve of the electrolytic cell and the actual power absorption curve of the super capacitor in the whole process, as shown in figure 10. It should be noted that for the sake of description, the state of zero absorption power of the cell in Figure 10 is that the cell operates under the condition of thermal insulation power by default.

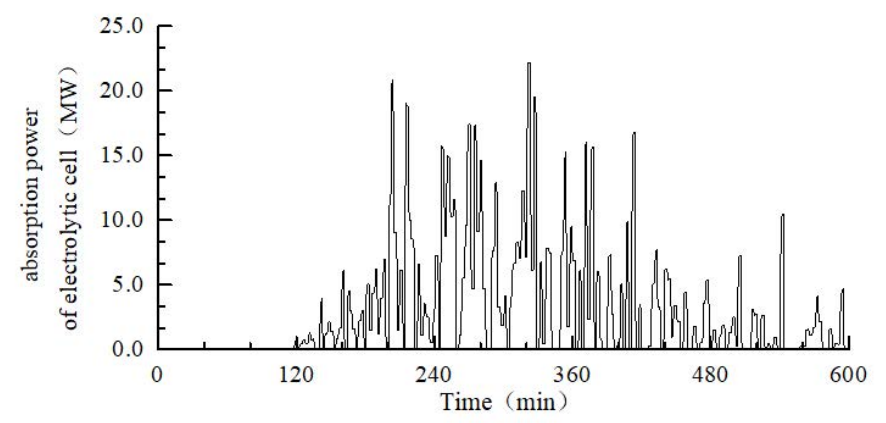

(a) Power absorption curve of electrolyzer

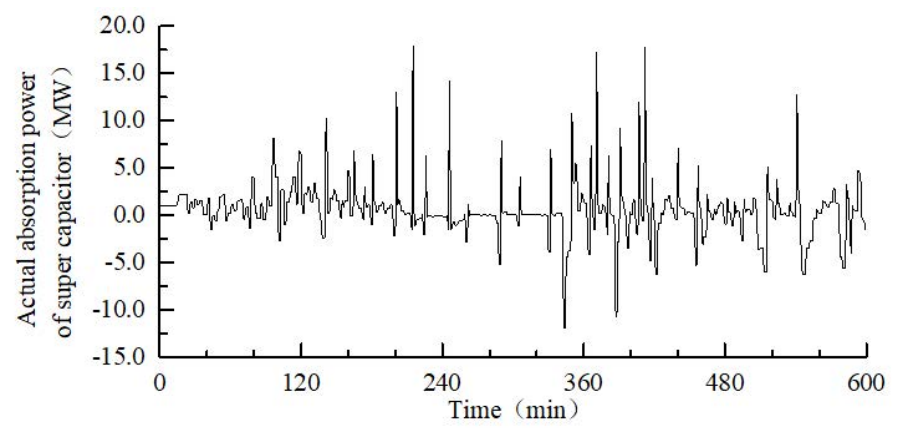

(b) Power absorption curve of super capacitor

Fig.10. Electrolyzer and Super capacitor power absorption curve

According to figure 10 (a), the capacity of the electrolytic cell is $20 \mathrm{MW}$, and the corresponding cost is the maximum $F_{E L \max }$. As the main energy storage device, it absorbs most of the fluctuating power of wind power. As the auxiliary energy storage device, the super capacitor absorbs the high-frequency component of wind power and provides the ability to output power to the grid. Figure 11 shows the actual storage capacity of the super capacitor. It can be seen that its state of charge remains stable after saturation, ensuring the power output capacity of the energy storage system to the grid in the low wind power period. 


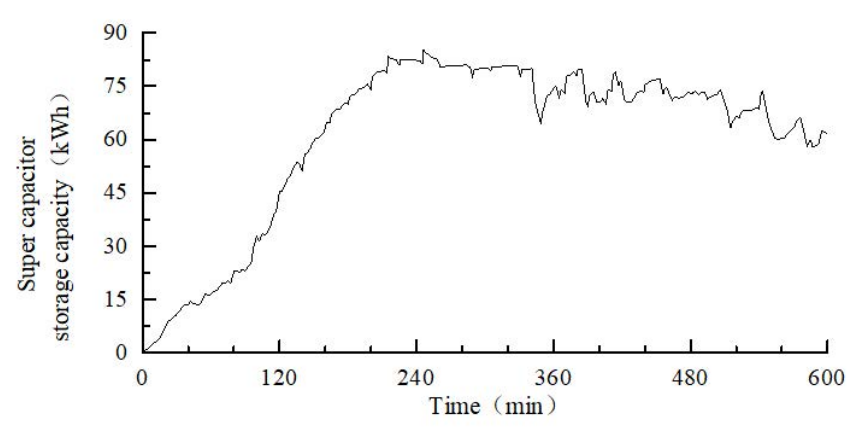

Fig.11. Super capacitor actual storage power change

\subsection{Configuration result analysis}

After the energy storage system is added, that is, after the low-pass filtering method is adopted, the wind power is divided into two parts: high-frequency and low-frequency, that is, the power that the energy storage system needs to absorb and the grid connected power. The grid connected power is shown in Figure 12. Compared with the original wind power, the added energy storage system can effectively reduce its volatility.

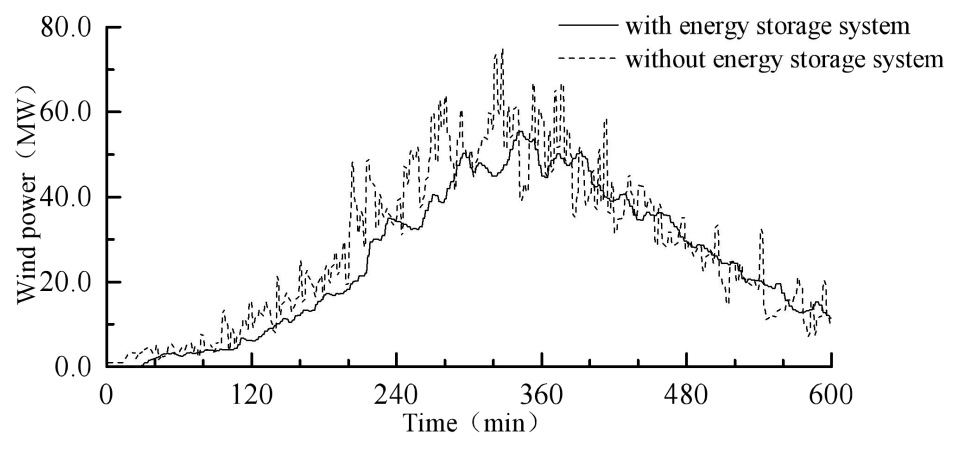

Fig.12. Grid-connected power waveform with or without energy storage system

Figure 13 is the evaluation result of the fluctuation of the stabilized grid connected power based on the window sliding observation method. According to figure 13, it is not difficult to find that the maximum value of $1 \mathrm{~min}$ fluctuation is $3.7 \mathrm{MW}$, which is less than $5 \mathrm{MW}$ of the national standard; the maximum value of $10 \mathrm{~min}$ fluctuation is $9 \mathrm{MW}$, which is less than $16.7 \mathrm{MW}$ of the national standard; at this time, the configured capacity is $20 \mathrm{MW}$ of electrolytic cell and $81.06 \mathrm{kWh}$ of super capacitor. Based on the configuration method proposed in this paper, the electrolyzer super capacitor hybrid energy storage system can effectively suppress the power fluctuation of wind power and achieve the expected goal. 


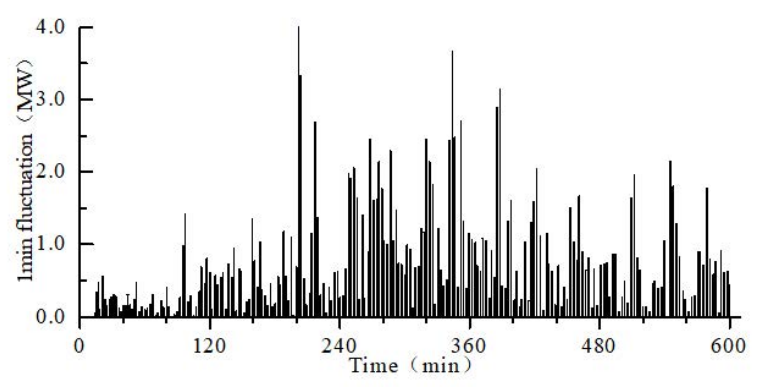

(a) 1 min fluctuation of grid connected power of energy storage system

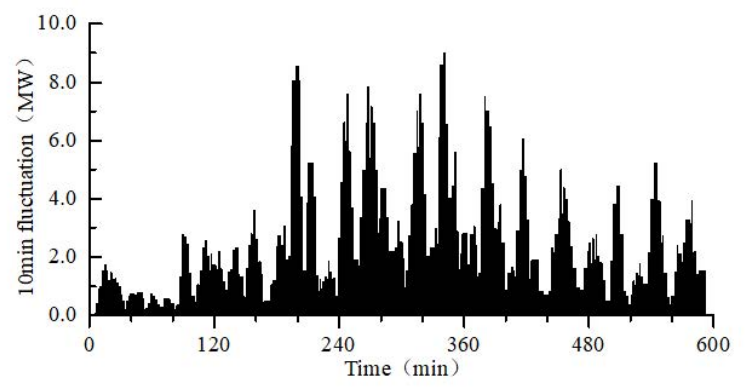

(b) 10min fluctuation of grid connected power of energy storage system

Fig.13. Fluctuation with the energy storage system

\subsection{Economy analysis}

First of all, for the wind farm, adding energy storage system will increase the disposable investment of the whole system, but it can make the output of the wind farm more stable and easier to connect to the grid. Practical experience shows that the energy storage system will increase the cost of wind farm by $3 \%$, but it can reduce $20 \%$ of the abandoned wind power.

In addition, the selection of energy storage equipment in the energy storage system is also an important factor affecting the economy. Compared with the traditional battery and super capacitor hybrid energy storage system, the combination of the electrolyzer and super capacitor hybrid energy storage system requires higher capacity of the super capacitor. This is because the electrolyzer can only absorb power but not release power, so the traditional energy storage system has more advantages in terms of disposable investment; for energy conversion efficiency, battery is the mutual conversion of electric energy [22]. The energy conversion efficiency of lead-acid battery is $80 \%$, while that of lithium-ion battery is $97 \%$. The energy conversion efficiency of the electrolyzer is about $55 \%$, which is also the disadvantage of the energy storage system.

However, compared with the battery, the cell has longer cycle life and lower 
maintenance cost, which is a better choice in the long run. More importantly, hydrogen is a kind of clean energy carrier with multi-purpose and high energy density. At present, hydrogen is mainly produced by chemical methods, which not only costs a lot but also pollutes the environment. If we use renewable energy to produce hydrogen, we can solve this problem well and make great contribution to the popularization of hydrogen energy. From this point of view, the energy storage system of electrolyzer has a more promising future.

\section{Conclusion}

As a solution to reduce wind power fluctuation, the wind hydrogen coupling system can not only improve the wind power and user friendliness, but also realize the green production of hydrogen, which has a good application prospect. The main conclusions are as follows:

1) A window sliding observation method of wind power fluctuation is proposed. By combining with low-pass filtering method, the quantitative evaluation of wind power fluctuation is realized intuitively.

2) Based on the characteristics of electrolyzer and super capacitor, a low-pass filter configuration method and flow chart of hybrid energy storage system are proposed. This method can realize the minimum initial investment cost under the condition of meeting the national grid connection standard.

3) The rotation optimal control strategy of the electrolytic cell and the coordinated control strategy of the cell and super capacitor hybrid energy storage system based on the SOC of super capacitor are proposed. The work of cell array equalization is realized and the power output capacity of super capacitor to power grid is guaranteed.

4) The simulation results show that the proposed hybrid energy storage system configuration method and control strategy are effective, which can effectively reduce the fluctuation of wind power grid connected power to meet the grid connected standards.

\section{References}

1. M. Ni, M. K. H. Leung, K. Sumathy, E. C, 18(5), 5-9, (2004)

2. M. Korpas, A. T. Holen, IEEE Trans Energy Convers, 21(3), 742-749, (2006)

3. Z. Z. Chen, E.E, 1, 12-14, (2001)

4. C. X. Lü, X. R. Li, L. H. Hu, Automat Electron Power Sys, 2, 21-29, (2015)

5. L. Lin, L. Zhu, R. Yang, Y. Gao and Q. Wu, ICPRE, 61-65, (2017)

6. G. W. Cai, C. Chen, L. G. Kong, Power Sys Techno, 40(10), 2982-2990, (2016) 
7. X. F. Men, Y. Luo, O. D. Crisalle, Renew Energy, 34(11), 1595-1602, (2016)

8. T. Zhou, B. Francois, IEEE Trans Ind Electron, 58(1), 95-104, (2010)

9. J. C. Zhao, J. Chen, Y. X. Wang, PCRC, 38(6), 125-129, (2017)

10. G. W. Cai, L. G. Kong, A. Z. Xu, T China Electro Soc, 20, (2017)

11. G. W. Cai, L. G. Kong, Y. Xue, Automat Electron Power Sys, 21, 127-135, (2014)

12. J. Lei, Q. Gong, International Journal of Energy Research, (2018)

13. X. J. Shen, X. Y. Zhang, T. T. Lie, Int J Energ Res, 42(10), 3244-3257, (2018)

14. S. M. Schoenung, W. V. Hassenzahl, (2007)

15. Y. Z. Lei, W. S. Wang, Y. H. Yin, Pro CSEE, 22(5), 32-35, (2002)

16. GBT_19963-2011, technical regulations for wind farm access to power systems

17. D. W. Huang, G. W. Cai, D. F. Yang, et al. Acta Energi Sin

18. W. B. LI, J. P. LU, B. XU, ECEP, 3, 439-444, (2012)

19. X. Q. Huang, R. X. Ding, W. Xiao, Elec Power Cons, 35(12), 67-71, (2014)

20. D. D. Li, C. Chen, Pro CSEE, 25(21), 41-44, (2005)

21. M. Asmine, J. Brochu, J. Fortmann, IEEE Trans Power Sys, 26( 3), 1769-1782, (2011)

22. Z. L. Zhang, Y. Y. Cai, Y. Zhang, IEEE Trans on Power Elec, 31(1), 1-1, (2015) 